\title{
Nursing Information Flow in Long-Term Care Facilities
}

\author{
Quan Wei ${ }^{1}$ Karen L. Courtney ${ }^{2}$ \\ ${ }^{1}$ Alberta Health Services, Calgary, Alberta, Canada \\ ${ }^{2}$ School of Health Information Science, University of Victoria, \\ Victoria, British Columbia, Canada \\ Address for correspondence Quan Wei, MSc, MBA, Alberta Health \\ Services, 92 Chaparral Way SE, Calgary, Alberta T2X 3J9, Canada \\ (e-mail: Jen.wei@ahs.ca).
}

Appl Clin Inform 2018;9:275-284.

\begin{abstract}
Keywords

- electronic health records

- nursing informatics

- Canada

- skilled nursing facilities

Objective Long-term care (LTC), residential care requiring 24-hour nursing services, plays an important role in the health care service delivery system. The purpose of this study was to identify the needed clinical information and information flow to support LTC Registered Nurses (RNs) in care collaboration and clinical decision making.

Methods This descriptive qualitative study combines direct observations and semistructured interviews, conducted at Alberta's LTC facilities between May 2014 and August 2015. The constant comparative method (CCM) of joint coding was used for data analysis.

Results Nine RNs from six LTC facilities participated in the study. The RN practice environment includes two essential RN information management aspects: information resources and information spaces. Ten commonly used information resources by RNs included: (1) RN-personal notes; (2) facility-specific templates/forms; (3) nursing processes/tasks; (4) paper-based resident profile; (5) daily care plans; (6) RN-notebooks; (7) medication administration records (MARs); (8) reporting software application (RAI-MDS); (9) people (care providers); and (10) references (i.e., books). Nurses used a combination of shared information spaces, such as the Nurses Station or RNnotebook, and personal information spaces, such as personal notebooks or "sticky" notes. Four essential RN information management functions were identified: collection, classification, storage, and distribution. Six sets of information were necessary to perform RN care tasks and communication, including: (1) admission, discharge, and transfer (ADT); (2) assessment; (3) care plan; (4) intervention (with two subsets: medication and care procedure); (5) report; and (6) reference. Based on the RN information management system requirements, a graphic information flow model was constructed.

Conclusion This baseline study identified key components of a current LTC nursing information management system. The information flow model may assist health information technology (HIT) developers to consolidate the design of HIT solutions for LTC, and serve as a communication tool between nurses and information technology (IT) staff to refine requirements and support further LTC HIT research.
\end{abstract}




\section{Background and Significance}

Long-term care (LTC) refers to residential care or complex care, where care is provided to residents (such as seniors and people with disabilities), who have a limited ability to direct their own care and require 24-hour nursing services in facility-based care settings. ${ }^{1}$ Currently, LTC services face substantial population health challenges with increasing demands for highquality services and capacity. Worldwide, the population is aging and living longer than past generations, and therefore, more care will be required. According to a Statistics Canada report, ${ }^{2} 7.1 \%$ of the almost 5 million seniors aged 65 and older live in care facilities and the proportion of these seniors will increase to approximately one-quarter (25\%) of the population over the next few decades.

Care collaboration becomes an essential aspect of nursing care in LTC settings. Registered Nurses (RNs) play leadership roles in care collaboration and they face increasing challenges with clinical information communication in a timely manner because of the limitation of technology tools and provider-toRN communication. There is a rapid rise in the need for efficient clinical information sharing across care settings. In Canada, LTC has been included in numerous large-scale health information technology (HIT) initiatives and implementation plans. ${ }^{3,4}$ But there have been increasing concerns about the effectiveness of current information technology (IT) solutions used in LTC facilities. Challenges in using software to improve the efficiency of clinical processes were repeatedly reported in outcome evaluation studies in some LTC facilities. ${ }^{5-7}$

Meeting user requirements is critical to the success of a system development and usefulness. The successful compilation and execution of the implementation is dependent on well-documented user requirements specification containing clear, concise, testable, and traceable requirements. Usercentered design requires an analysis based on users' information management activities; an understanding of the needs for information; and streamlined information processing flow from systematic point of view. Both information flow and content requirements are major characteristics considered during information system development. The information flow path ensures information processing within the system with less interruption; the content requirements should describe the need that users must have in a system. On the other hand, information flow processes should describe how the required information flows upstream and downstream in a system to support user performance. Research has shown that information flow analysis is an effective method used for understanding user perspectives toward needs regarding HIT design and implementation. ${ }^{8-14}$

In the evidence to date, LTC HIT has both positive and negative effects on nursing practice and care quality. ${ }^{5,6,12,15-17}$ The careful consideration of the needs of nursing practice specific to the LTC setting was important and is addressed by the majority of the studies. Prior work ${ }^{5,12}$ identified the complex nature of care collaborative work, the layers of information exchange for the delivery of quality, the diverse number of internal and external communication channels, and artifacts used in LTC settings. There was little description of all-inclusive or end-to-end RN-focused information flow or RN requirements. Discussions associated with user requirements can be found in three main areas: (1) information process flow challenges in relation to nursing practice; $6,7,14,18-20$ (2) information requirements for information quality; $;^{21-24}$ and (3) communication demands for care collaboration. $5,12,15,17,25-27$

The need of comprehensive communication has been well addressed by the majority of reports as LTC care requires increased collaboration., 5,12,18,26-28 Care collaboration requires extensive information sharing and communication, which can challenge the design process to achieve fewer interruptions in information flow processes. "One of the barriers to information communication technology (ICT) diffusion in aged care is the failure to cater for the complex and interdisciplinary requirements of the aged care environment" Georgiou et al (p. 770). ${ }^{5} \mathrm{RN}$ information communication is facilitated by various information resources and information processes within existing information management structures. ${ }^{5,7,12,29}$

Some studies indicated that the physical aspects of workplace environment factors could have a direct effect on user information flows because those factors may influence user practice patterns in terms of information management activities, depending on where and how information is stored and exchanged. Thus, environment factors should be considered during a HIT design. 5,12,14 Tang and Carpendale ${ }^{10}$ identified three key environmental factors to consider in designing a system, including media (paper-based, verbal, displayed, and digital sources), common information space (shift change room, the nursing station, and the computer terminals), and personal information space (personal note, stickers). However, the HIT studies conducted in LTC settings mainly focused on a single care process, such as medication administration process. ${ }^{12,17,20,30}$ The literature review demonstrated that a baseline study was necessary to fulfill the gaps in the lack of documentation of RN information flow and information needs in current LTC settings. This study examined the RN perspectives to map a baseline RN information flow model. The model was based on RN information management activities with regard to information requirements within existing information resources and physical working environments. Thus, the goal of this investigation was to illustrate an end-to-end RN information flow including required information content.

\section{Objective}

The purpose of this study was to identify the necessary clinical information and information flow needed to support LTC RNs in care collaboration and clinical decision making. The intended output of this study was an information flow model that matched the scope of $\mathrm{RN}$ practice and nursing processes in the context of Canadian collaborative LTC environments.

\section{Methods}

\section{Study Design}

A qualitative research methodology was chosen for this study. Qualitative data were collected through direct 
observations and semistructured interviews of RN participants in LTC. Because our intended deliverable was an information flow model for RN practice, the five-step nursing process model (assessment, diagnosis, planning, implementation, and evaluation) was used as a foundational framework in data collection.

Collected data were analyzed through the constant comparative method (CCM) based on the grounded theory methodological framework developed by Glaser and Strauss ${ }^{31}$ and Lincoln and Guba. ${ }^{32}$ The inductive data coding process allowed newly collected data to be compared with previous collected data. Analysis continued until data saturation. A visual representation of RN information flow was developed based on the categories and relationships that emerged from the data analysis.

\section{Recruitment}

The recruitment involved two steps: facility selection and RN participant recruitment.

\section{Facilities}

This study was initiated in six LTC nursing homes in Alberta, Canada. Publicly listed LTC facilities were considered for inclusion. The site recruitment process was facilitated by the leadership in two provincial organizations, Alberta Health Services and Covenant Health of Alberta. The selected facilities were based on facility operational and administrative approvals for the study, clinic accessibility, and interested, qualified RNs. The LTC facilities without RNs onsite were excluded from this study. Six LTC facilities, including publicly funded, nonprofit, and for-profit facilities, participated in this study. At a minimum, all had basic electronic access to the Resident Assessment Instrument Minimum Data Set (RAI-MDS). Across the sites, three different software vendor products were present. The use of e-documentation, or documentation of the care record electronically, varied from facility to facility.

\section{Participants}

Nine RN participants were recruited from nine different work sites in six nursing homes. The selection of eligible RN participants was based on the three criteria: (1) current $\mathrm{RN}$ registration; (2) provided direct care in nursing homes in Alberta; and (3) a willingness to participate. Written, informed consent was received from participants prior to the investigation; verbal consent was obtained from the resident if a resident could be observed as part of the nursing observation. No residents refused the observation.
The RN participants in the study had a median age of 41.5 years. The study included seven females $(78 \%$ ) and two males $(22 \%)$. The nurses had a median of 7.8 years LTC nursing experience and a median clinical nursing practice experience of 15.10 years. Seven participants were bachelor prepared RNs, one was the equivalent of an associate degree prepared nurse, and one had a PhD in nursing following foreign nursing preparation.

\section{Direct Observation}

A total of 18 hours of direct observations took place during the RN participants' shifts at their work sites. This observational sampling structure sought to fully represent the range of activities at different RN shifts (morning, afternoon, and evening) and different work sites (or floors or wings). An observation tool was developed to guide and record the observations and notes were taken throughout the observations.

Details recorded included: the types of information or data to be checked; data sources and the locations; nursing tasks and nursing processes; activities and related clinical functions; information hand-overs; and information media used to facilitate communication.

\section{Semistructured Interviews}

After the observation, each participant was interviewed using a semistructure interview guide for $\sim 30$ minutes and audiotaped for later transcription and analysis. The purpose of interviews was to confirm observations and understand the information content required for carrying on the care activities, including information sharing and communication. Nurses were also asked for self-reflection on their information management practices.

\section{Nursing Documentation Forms}

Blank nursing care forms were collected from each site to capture additional information on the nursing tasks and the task-related data that may not have occurred during the observations. A list of RN tasks was recorded in an MS Excel spreadsheet and verified by the participants.

\section{Data Analysis}

The inductive data analysis of the observations and interviews proceeded in four steps: (1) segmentation and coding; (2) category creation; (3) concept forming, and (4) model establishment ( - Fig。 1$)$. Key words were highlighted for distinctive meaning and a code (label) was attached to it at

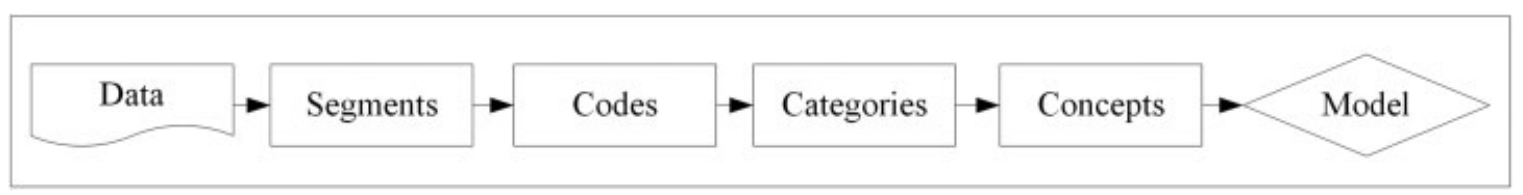

Fig. 1 Data analysis process. 
step 1. Codes were constantly compared on similarities, differences, and relationships. Codes that were similar to each other became clustered together as a category at step 2 . Nursing tasks within each category were revalidated with participants as necessary for their practice.

Similarly, when there were differences in meaning with existing categories, new categories were created. In step 3, concepts were formed from merging of the categories, based on the relationships between categories. Visual representations of the RN information management activities and the $\mathrm{RN}$ information flow were mapped to a model, based on the concepts that emerged from the observational and interview data.

\section{Results}

\section{Site e-Documentation Characteristics}

The self-reported percentage of e-documentation to complete required nursing documentation ranged between 10 and 95\%. Participants at the lowest reported usage were RNs limited to basic RAI-MDS reporting as e-documentation. Participants at the highest reported usage were able to utilize e-documentation for almost all of their documentation. No RNs reported $100 \%$ rates for e-documentation as all sites required some paper-based documentation, such as a medication administration record (MAR) and a paper-based resident profile for use by external health care providers. Nonetheless, several RNs reported that the e-documentation saved them tremendous time due to less handwriting; in contrast, other RNs commented that they must take additional time to transfer information from paper-based resident charts onto an RAI-MDS system to submit the reports. While the nursing clinical practice processes were consistent across sites; facilities with less functionality for e-documentation had more information resources and spaces for RNs to navigate in their information management processes. In comparing the facilities, the nonprofit facilities were more likely to have higher levels of technology usage and higher levels of IT integration in care management processes.

\section{Information Communication Strategies}

$\mathrm{RN}$ practice drove information management approaches to information capture, documentation, analysis, sharing, and distribution for resident care. Under the collaborative care approach and with limited IT resources in place, RNs creatively utilized different strategies to communicate clinical information in writing via paper, electronic means, and displays. The electronic communication encompassed any electronic media content that was intended to be accessed by RNs in either an electronic form or as printed output such as e-documentation, email, or fax. Both email and fax were regularly used tools to support RN communication internally and externally.

The paper-based communication method was preferred by most RNs and included (1) resident profile; (2) policy and procedures manuals; (3) RN-notebook; (4) printout forms; and (5) personal notes. A paper-based resident profile included information about care activities such as care plans, progress notes, MARs, and resident conference records. Because the resident profile had contributions from all internal and external providers, it was considered the most trustworthy information source by nurses. Handwritten RN-notebooks were observed at RN desks in each of the study sites. A handwritten RN-notebook was called "the Bible" by several RNs to indicate its importance for storing information and to keep track of tasks and share with other RNs. Usually, an RN would review the notes left by the previous RN, and then created a new "to-do list" as followup tasks in the notebook at the beginning of the shift and would check it regularly throughout the shift. Additionally, some RNs preferred to write shift summaries down in the RN-notebooks for shift reports. At some sites, shift summaries were printed out, but most RNs still wrote shift summaries in RN-notebooks. The RN-notebook served as a key platform for RN-to-RN communication. Several RN participants indicated that they relied on handwriting "for almost everything." The most common forms of handwritten documentation were care plans, progress notes, MARs, and resident conference records.

Frequently, RNs used personal notes to track information for their own use. Several RNs also had their own personal notebooks to record information at points of care, separate from the RN "Bible" notebooks. Collected information was later transferred into resident profiles (electronic or paper) after the RN returned to the nursing station. Some RNs expressed that they felt secure when they had a paper-based documentation on hand because they could find information more easily than in a computer system.

Display media, such as white boards (or information boards) and paper posters, were easy to observe at facilities and mainly used for internal communication.

Two types of verbal communication were identified: telephone and face-to-face. Direct verbal communication was considered the best choice, acknowledged by most of RNs. RNs made phone calls frequently to reach both internal and external providers. The telephone was used as a primary tool for external communication, such as contacting physicians, pharmacists, and scheduling external referrals.

\section{Information Spaces}

Information spaces were physical locations where information exchanges occurred inside facilities. Information exchange at different locations could alter the flow of information. The two types of information spaces were shared versus personal. A shared information space allowed multiple users to access the same information. For example, a nursing station was a typical shared information space where all RNs were able to access computers, resident profiles, reference books, telephones, or fax machines. In contrast, a personal information space specified a place to store information for individual use only. For instance, a personal notebook or a sticky note, which was used commonly by RNs to take notes during a procedure or an assessment at a resident's bedside. 


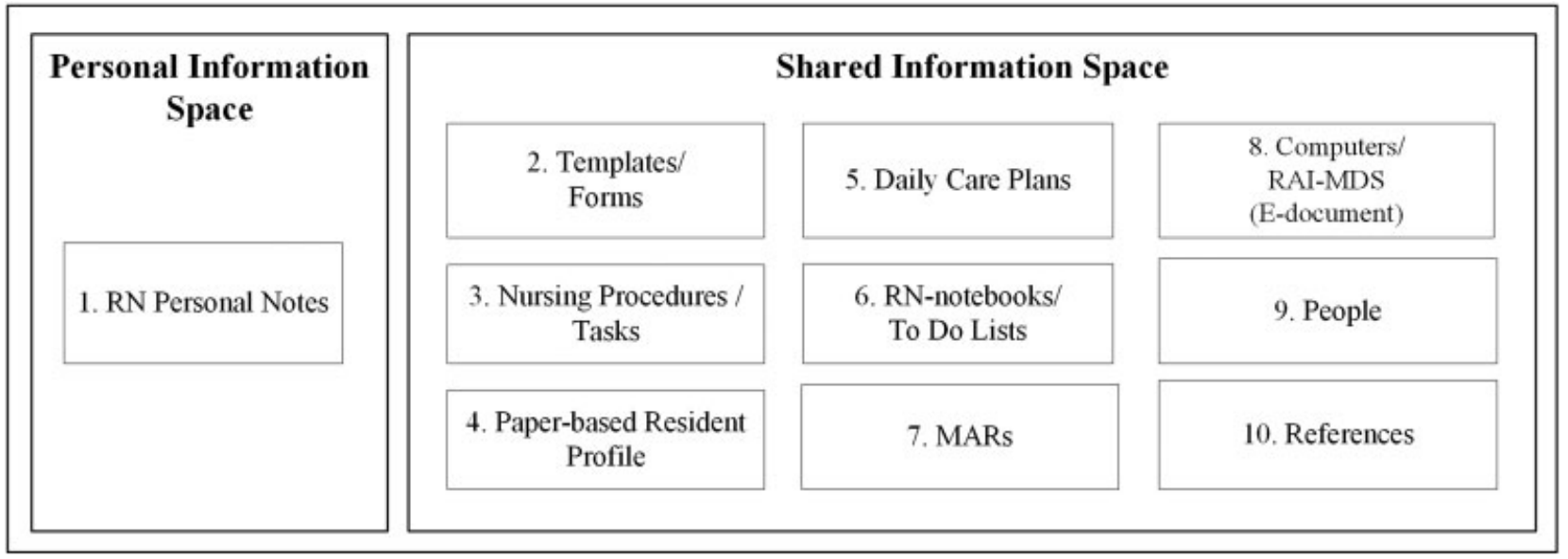

Fig. 2 Common information resources note. The number of information resources may be different depending on how information resources were organized.

\section{Information Resources}

Information resources indicated the sources of information used for generating knowledge. For example, a care plan outlined the plan of care and was an important information resource for RNs to guide care. Ten essential information resources were identified and had a unique physical location within the LTC facilities (-Fig. 2). An information resource might contain several different types of information content.

When RNs had more information resources, they had to move more to access information. Despite the variety of information resources that RNs needed to access, participants repeatedly mentioned a few specific content needs that were essential for e-documentation of their practice: MARs; immunization management; and consent management. For these items, the RNs preferred electronic documentation.

\section{Information Management Activities}

RN information management activities relied on their responsibilities to capture current clinical data and share information with multidisciplinary care team for delivering holistic care. RN information management activities occurred at each step of the nursing process. The nursing process is a five-step, information-driven process, from assessment to diagnosis, planning, implementation, and evaluation. ${ }^{33}$ By following the nursing process, RNs generated knowledge and made care decisions.

In - Fig. 3, key activities were structured into swim lanes to show the relationship between nursing activities, information resources, and spaces. There was repetition of documentation across various information resources. For example, an RN documented giving medications on the MAR and in the RAIMDS system; and updated the RN-notebook, the care plan, and the paper-based resident profile.

\section{Information Content Requirements}

In practice, RNs performed many different types of care tasks and each may require different types of information content. Eight task-related content types and one additional nontask- related category, references, were observed. References designated the collection of nonresident-specific information materials such as policy manuals. Through merging the content types, six categories of RN information content requirements were derived ( - Fig. 4): (1) administration/ discharge/transfer (ADT); (2) assessment; (3) care plan; (4) intervention (medication and care procedures); (5) report; and (6) references. Each category contained multiple levels of subcategories of information content ( - Fig. 4).

\section{Information Flow Model}

The information flow model was derived from a constant comparative process involving observation and interviews with participants across work settings, available IT, and communication activities.

The model used an agnostic IT approach; therefore, the model itself does not specify if a particular information resource is paper or electronic based. Because all of the participants in the study were providing direct care to residents, the model also reflected a hands-on, provision of care type of nursing practice and does not reflect any administrative roles nurses may also have had.

This model (-Fig. 5) demonstrated the RN information flow, including required functions, information sets, nursing processes, and care collaboration.

Four information management functions were fundamental to support RN practice: collection, classification, storage, and distribution.

Collection: The collection function was linked to the information generated by nursing tasks (ADT, assessment, care plan, and intervention). Examples of nursing collection functions include taking notes, checking references, or participating in a care conference. The reference and report categories also contributed to the collection because the feedback of information (i.e., a clinical report) was important for assisting RNs in clinical decision making.

Classification: The collected information was then classified (analyzed and interpreted) by the nurse and used to 


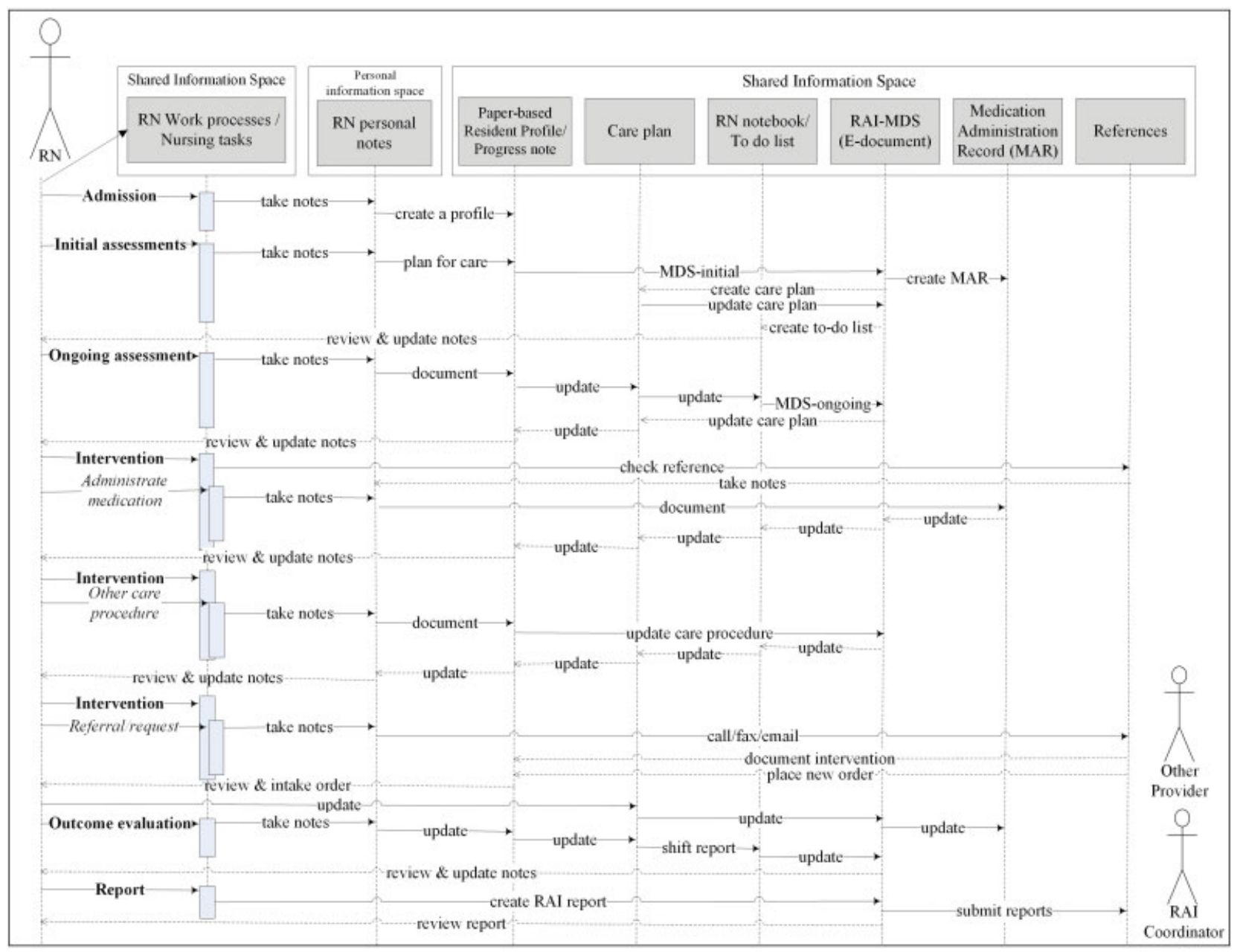

Fig. 3 Registered Nurse (RN) information management activities and interactions.

make clinical decisions and new reports. The reference and report content categories informed the classification process. Nurses drew on previously reports of residents and reference materials to give context to newly collected information. An example of a nursing classification function would be the production of a RAI-MDS report or a shift report.

Storage: Clinical documentation for nurses was both a professional and legal responsibility. Clinical decisions (i.e., renew a care plan) or clinical outcomes were stored (documented) on the resident profiles either on the paper-based binders or in the RAI-MDS applications.

Distribution: Distribution was the sharing of clinical documentation with other providers. Distribution as a function relied on the report information category. Examples of nursing distribution include making referrals to other health care providers or sending a medication list to a pharmacy. Care collaboration was a key goal of the distribution function. The lack of a shared information platform with external health care providers resulted in inconsistent clinical information and consequently may have resulted in additional time spent for planning care and gathering and organizing information. In turn, this resulted in clinical inefficiency.

The RN information flow model incorporated the six information content sets and multiple subsets to represent the movement of information. The nursing process (assessment, diagnosis, planning, implementation, and evaluation) is informed by the four information management functions (collection, classification, storage, and distribution). Information content categories were represented in multiple information management functions reflecting the iterative nature of clinical information. Care collaboration was demonstrated through the distribution and sharing of information with internal and external providers.

\section{Summary}

-Fig. 5 also represents a current RN information management system with all of the components of the RN requirements described above. This figure highlights the four required information management functions; the six required information content categories; practice requirements of nursing process; integrated care collaboration; and information flow directions.

\section{Discussion}

This study is one of the few studies assessing nursing requirements and end-to-end information flow in LTC settings. Similar to this study, several other studies observed a 


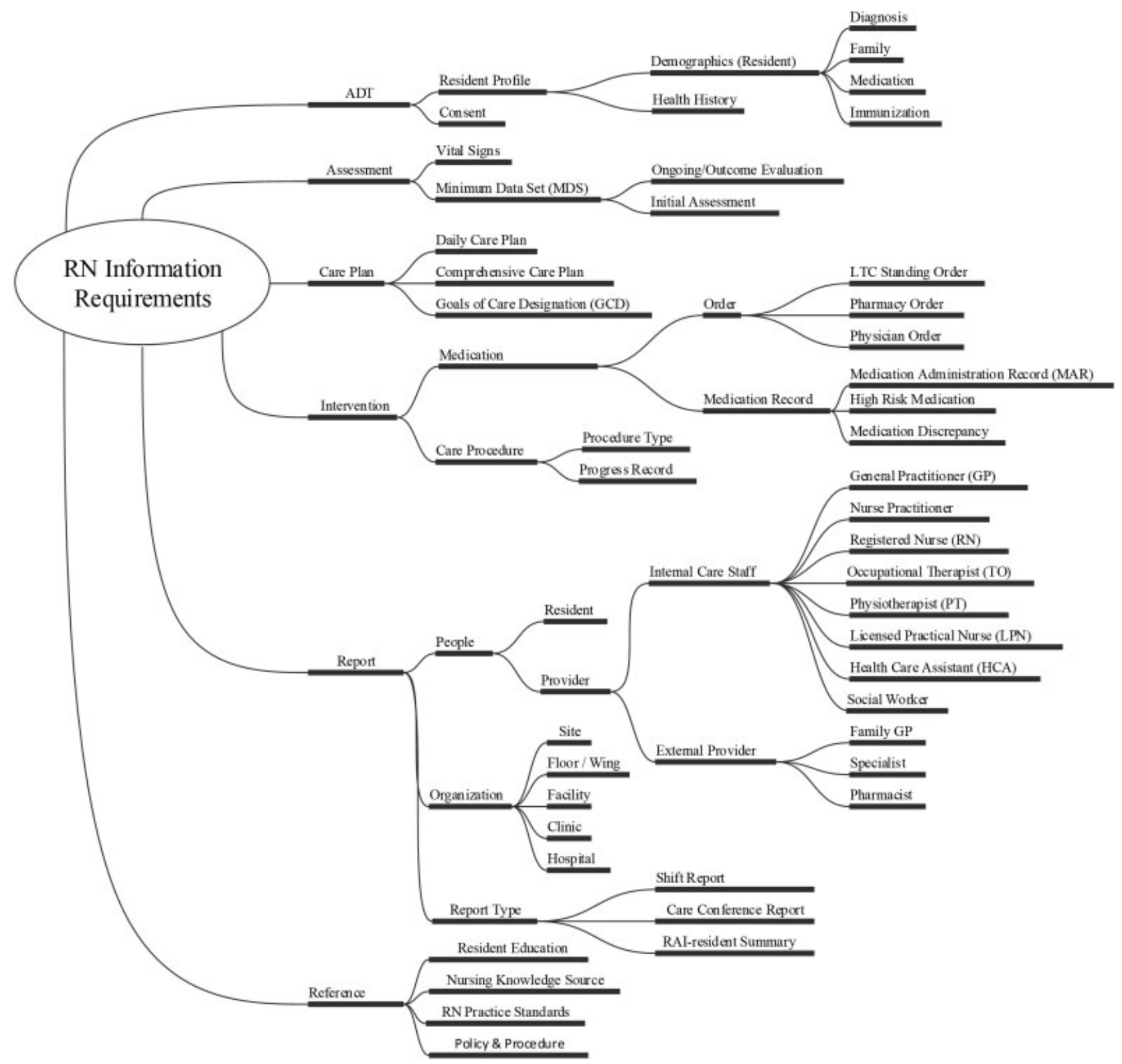

Fig. 4 Information sets and subsets.

lack of IT to support clinical work ${ }^{34,35}$ and demonstrated complex, interruption-oriented communication patterns in LTC settings. $5,12,15,26,27$ In this study, care collaboration is managed through multiple layers of operations across different care settings, such as acute care hospitals, primary care clinics, public health clinics (i.e., immunization), and palliative care for end-life care. Information flow outside of the facility was outside the scope of this study and is a limitation. Further work examining the information across transitions of care (leaving and returning to LTC) is needed and may yield additional findings for required functionality and unique content. Other researchers have noted that the importance of health information exchange in LTC is expected to grow with an increased demand for information systems to support clinical processes. ${ }^{36,37}$

This study did not compare resident outcomes related to e-documentation rates or available information system func- tions. There are examples in the literature of no improvement in resident outcomes following HIT implementation. ${ }^{38}$ A recent systematic review focusing on resident quality outcomes associated with electronic health records in LTC had mixed findings. ${ }^{39}$ While this study sheds light on common content and information workflow across several LTC facilities, it does not replace the need for a facility-level assessment of information needs and workflow for clinical care prior to information system implementation. This study may inform and reduce the time needed for a local needs assessment.

Or et $\mathrm{al}^{6}$ reported that poor usage of clinical information systems in nursing homes was the result of the system not matching nurses' needs. This pattern is consistent with the outcomes of this study. This study found substantial variation (10-95\%) in electronic documentation. All RN participants, particularly the RNs with lower usage, expressed 


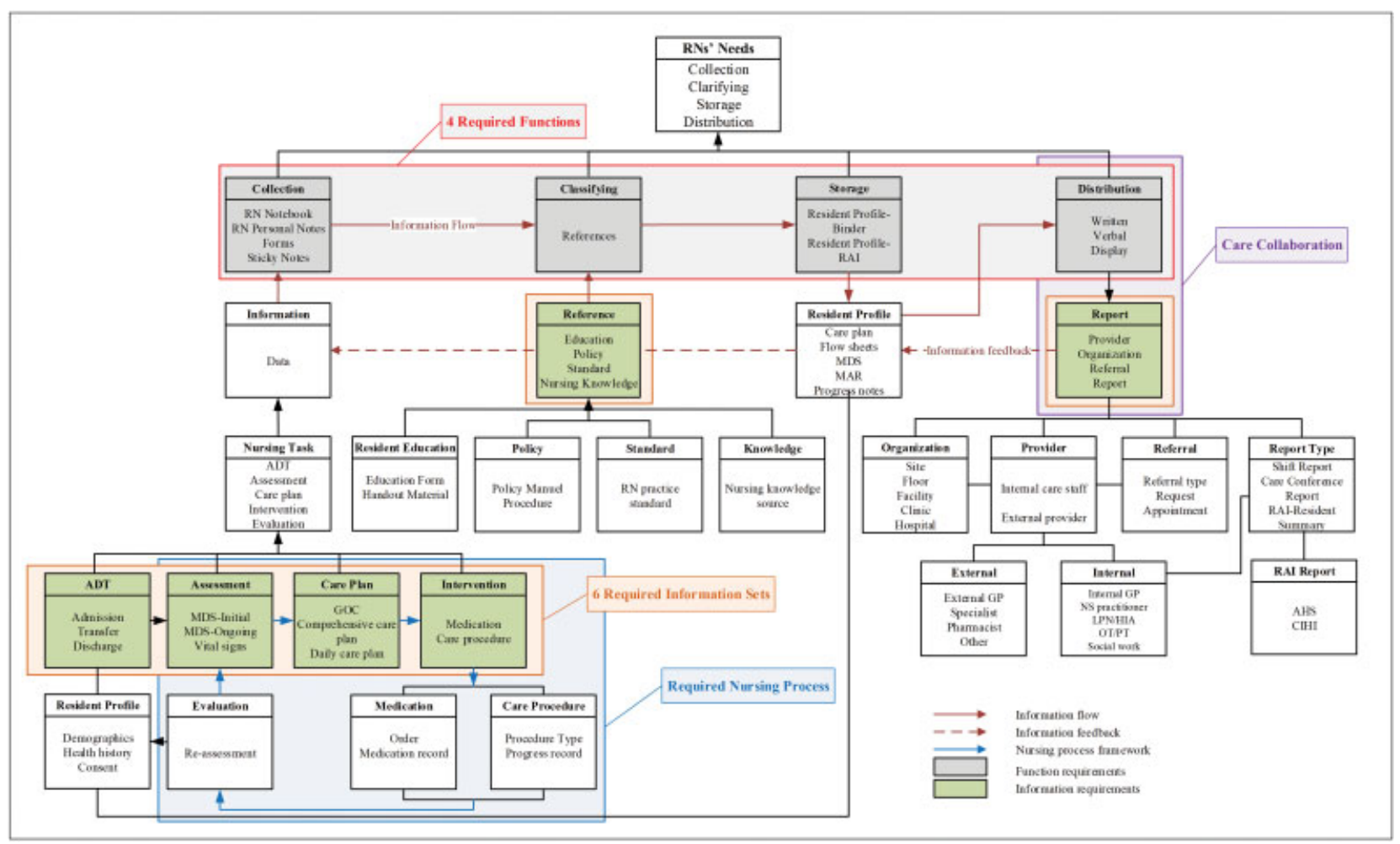

Fig. 5 Registered Nurse (RN) information flow model.

eagerness to use more electronic information management options if the technology could better support their needs. Additionally, several RNs wished that the RAI-MDS could have better features for resident assessments. For example, after performing a fall assessment on a resident, a nurse will enter a score into the RAI-MDS tool. However, there is no space to document clinical findings. This is important because RNs need to know the clinical explanation for the unusual score. Searching for this explanation adds additional tasks and nursing time; contributing to clinical inefficiency and patient safety issues.

Furthermore, RNs expect better integration between technology applications and other electronic devices, such as printers and fax machines. Some RNs mentioned that although they can use e-document for "almost everything," they could not print out a referral form or transfer form directly with the resident's demographic information on the form. Thus, RNs had to still fill out the form by hand. Likewise, record inaccessibility and the lack of ability to document at the point of care perpetuate the use of personal, handwritten documentation and process work arounds. Alexander et al's study ${ }^{40}$ suggested that mobile technologies might be the most useful solution in meeting this accessibility gap. Lack of technology integration and limited clinical support have real-world consequences for residents. ${ }^{34,35,41}$ In the United States, increased sophistication of IT capabilities, use, and integration was associated with decreased deficiency scores in nursing homes. ${ }^{35}$ Similar studies of Canadian LTC facilities would be useful.

In this study, not-for-profit nursing homes tended to have higher levels of technology use and integration of those systems into resident management processes. This finding is consistent with Alexander et al's ${ }^{42}$ study. This study observed facilities with different types of ownership, further studies in other provinces and countries with other payor systems are needed to see how ownership may affect IT investment and adoption.

This study produced observational findings of nursing in six nursing homes in Alberta. It has a limited sample of LTC facilities, which may not be generalizable to other settings. Further research of other user groups in LTC can contribute to improving the design of HIT systems to produce further gains for LTC facilities.

\section{Conclusion}

This study identified major influencing factors in current RN information management system as well as a visual information flow model with RN required components. The RN information flow model offers a baseline that may assist analysts and HIT development teams to further prioritize, organize, and validate requirements as well as transform the baseline data into system requirements for HIT solutions in LTC. The findings also indicated that the HIT adoption in LTC settings needs to be tailored for the complex and multidisciplinary requirements for care collaboration.

As an early investigation on RN requirements in LTC setting, this study specifically focuses on direct care RNs with no predefined requirements. Demographic characteristics may have influenced RN perspectives toward technology and nursing practice. However, the categories in the RN 
tasks could assist a developer in specifying the information requirements to represent commonly needed information content by RNs for clinical practice in the LTC setting.

$\mathrm{RN}$ responsibilities may be facility- or jurisdiction-specific. The size of the facility, the number of RNs available, the RN-to-LPN ratio, and the number of residents could affect the $\mathrm{RN}$ role and/or tasks. For example, the study found that some RNs not only provided direct resident care, but also performed duties as managers.

This study brings together work in key areas of RN requirements. To support HIT adoption in LTC settings, more research is needed for user requirements, mechanisms for technology integration, and health information exchange with external applications. Other nursing user groups, such as LPNs and health care or nursing assistants, should be considered because these user groups are not only data gatherers, but data users. Information system integration with fax machines will engage users for LTC technology adoption. LTC application links to electronic medical record (EMR) systems will allow to share clinical information with external providers. Therefore, HIT design and implementation must consider all users' needs to build a shared information management platform for all providers who provide services to LTC.

\section{Clinical Relevance Statement}

This information flow model of a RN information management system in LTC could contribute a useful reference for HIT system design. The four information management functions provide high level functional requirements, while the information content categories could be used as a baseline reference of $\mathrm{RN}$ information requirements. These requirements could be used as a starting point for validation with local RNs prior to HIT design or implementation.

Diagramming the information workflows in this study also revealed that information systems with less functionality for e-documentation meant an increased number of physical information resources and spaces that nurses needed to consult in their daily care routine. Even without changing information systems, there may be gains in clinical efficiency through centralization of physical information spaces and reductions in the number of information resources that need to be used. Similarly, understanding the physical context of information resources is an important consideration for care collaboration with external health care providers.

\section{Multiple Choice Questions}

1. Which information resource was only found in the RN personal space?
a. Daily care plans
b. RN notebooks
c. RN personal notes
d. RN to-do lists

Correct Answer: The correct answer is option c, RN personal notes. Daily care plans, RN notebooks, and RN to-do lists may also be encountered within the personal spaces in LTC facilities, but they are not located exclusively within those spaces. The RN personal notes, which are not part of the formal patient record, are the only information resource that is exclusive to the personal space of the RN.

2. Care collaboration is an important activity for nurses in LTC. Which information management function is most associated with this nursing activity?
a. Collection
b. Classification
c. Storage
d. Distribution

Correct Answer: The correct answer is option d, distribution. Care collaboration often involves both internal and external care providers. The distribution function incorporates the idea of "pushing" information and data to other providers rather than waiting for those providers to access materials that have been "stored" (documented). As a driver of care collaboration processes, the distribution function best represents the push of information to other providers.

\section{Protection of Human and Animal Subjects}

This study was performed in compliance with the World Medical Association Declaration of Helsinki on Ethical Principles for Medical Research Involving Human Subjects and was reviewed and approved by University of Victoria Human Research Ethics Board (Protocol \# 13-447) and Human Research Ethics Board of Alberta - Community Health Committee (Protocol \# 14-0019). Organizational and administrative approvals were obtained from Alberta Health Services and Convenant Health.

\section{Conflict of Interest}

None.

\section{References}

1 Government of B.C. Long-Term Residential Care; 2017. Available at: http://www2.gov.bc.ca/gov/content/health/accessing-healthcare/home-community-care/care-options-and-cost/long-termresidential-care. Accessed April 16, 2018

2 Statistics Canada. Portrait of Families and Living Arrangements in Canada, Portrait of Families and Living Arrangements in Canada, families, households, marital status, 2011 census of population. Available at: http://www12.statcan.gc.ca/census-recensement/2011/ as-sa/98-312-x/98-312-x2011001-eng.pdf. Accessed April 16, 2018

3 AHS. Connect Care. 2018. Available from: https://www.albertahealthservices.ca/info/cis.aspx. Accessed April 16, 2018

4 eHealth Ontario. What we do; 2018. Available at: http://www. ehealthontario.on.ca/en/about-us/about-us/. Accessed April 16, 2018

5 Georgiou A, Marks A, Braithwaite J, Westbrook JI. Gaps, disconnections, and discontinuities-the role of information exchange in the delivery of quality long-term care. Gerontologist 2013; 53(05):770-779 
6 Or C, Dohan M, Tan J. Understanding critical barriers to implementing a clinical information system in a nursing home through the lens of a socio-technical perspective. J Med Syst 2014; 38(09):99

7 Yu P, Zhang Y, Gong Y, Zhang J. Unintended adverse consequences of introducing electronic health records in residential aged care homes. Int J Med Inform 2013;82(09):772-788

8 Alexander GL, Rantz M, Flesner M, Diekemper M, Siem C. Clinical information systems in nursing homes: an evaluation of initial implementation strategies. Comput Inform Nurs 2007; 25(04):189-197

9 Bayley KB, Savitz LA, Rodriguez G, Gillanders W, Stoner S. Barriers associated with medication information handoffs. Advances in Patient Safety: from research to implementation. Rockville, MD: Agency for Healthcare Research and Quality (US); 2005 Feb (3). Available at: https://www.ncbi.nlm.nih.gov/books/NBK20553/. Accessed April 16, 2018

10 Tang C, Carpendale S. An observational study on information flow during nurses' shift change. ACM Human Factors in Computing Systems; 2007 April 28-May 03 (1):219-228; San Jose, CA. Available at: http://innovis.cpsc.ucalgary.ca/innovis/uploads/Publications/Publications/paper823-tang.pdf. Accessed April 16, 2018

11 Tang C, Carpendale S. Supporting nurses' information flow by integrating paper and digital charting. ECSCW: Computer Supported Cooperative Work; 2009, September 07-11; Vienna, Austria:43-62. Available at: http://www.ecscw.uni-siegen.de/2009/ 6-Tang\%20and\%20Carpendale.pdf. Accessed April 16, 2018

12 Tariq A, Georgiou A, Westbrook J. Complexity of collaborative work in residential aged care facilities: an analysis of information exchange for medication management. HICSS; 2012; 45th, Jan 47. Hawaii:915-924. Available at: http://ieeexplore.ieee.org/document/6149006/?reload=trueat. Accessed April 16, 2018

13 Unertl KM, Weinger MB, Johnson KB, Lorenzi NM. Describing and modeling workflow and information flow in chronic disease care. J Am Med Inform Assoc 2009;16(06):826-836

14 Unertl KM, Johnson KB, Gadd CS, Lorenzi NM. Bridging organizational divides in health care: an ecological view of health information exchange. JMIR Med Inform 2013;1(01):e3

15 Cherry B, Carpenter K. Evaluating the effectiveness of electronic medical records in a long term care facility using process analysis. J Healthc Eng 2011;2(01):75-86

16 Kruse CS, Mileski M, Alaytsev V, Carol E, Williams A. Adoption factors associated with electronic health record among long-term care facilities: a systematic review. BMJ Open 2015;5(01):e006615

17 Yeh SH, Jeng B, Lin LW, et al. Implementation and evaluation of a nursing process support system for long-term care: a Taiwanese study. J Clin Nurs 2009;18(22):3089-3097

18 Brandeis G, Hogan M, Murphy M, Murray S. Electronic health record implementation in community nursing homes. JAMDA 2007;8(01):31-34

19 Tariq A, Lehnbom E, Oliver K, et al. Design challenges for electronic medication administration record systems in residential aged care facilities: a formative evaluation. Appl Clin Inform 2014; 5(04):971-987

20 Vogelsmeier AA, Halbesleben JRB, Scott-Cawiezell JR. Technology implementation and workarounds in the nursing home. J Am Med Inform Assoc 2008;15(01):114-119

21 Lindner SA, Davoren JB, Vollmer A, Williams B, Landefeld CS. An electronic medical record intervention increased nursing home advance directive orders and documentation. J Am Geriatr Soc 2007;55(07):1001-1006

22 Munyisia EN, Yu P, Hailey D. Does the introduction of an electronic nursing documentation system in a nursing home reduce time on documentation for the nursing staff? Int J Med Inform 2011; 80(11):782-792

23 Nahm ES, Mills ME, Feege B. Long-term care information systems: an overview of the selection process. J Gerontol Nurs 2006; 32(06):32-38
24 Ranegger R, Hackl WO, Ammenwerth E. Implementation of the Austrian nursing minimum data set (NMDS-AT): a feasibility study. BMC Med Inform Decis Mak 2015;15(01):75

25 Hustey FM, Palmer RM. Implementing an Internet-based communication network for use during skilled nursing facility to emergency department care transitions: challenges and opportunities for improvement. J Am Med Dir Assoc 2012;13(03):249-253

26 Nelson R. Connecting the providers in your healthcare community: one step at a time. Front Health Serv Manage 2005; 22(02):13-28

27 Alexander GL, Pasupathy KS, Steege LM, Strecker EB, Carley KM. Multi-disciplinary communication networks for skin risk assessment in nursing homes with high IT sophistication. Int J Med Inform 2014;83(08):581-591

28 Rantz MJ, Hicks L, Petroski GF, et al. Cost, staffing and quality impact of bedside electronic medical record (EMR) in nursing homes. J Am Med Dir Assoc 2010;11(07):485-493

29 Marquard JL, Mei YY. Using process visualizations to validate electronic form design. AMIA Annual Symposium Proceedings; 2010:497-501. Available at: https://www.ncbi.nlm.nih.gov/pmc/ articles/PMC3041292/. Accessed April 16, 2018

30 Pierson S, Hansen R, Greene S, et al. Preventing medication errors in long-term care: results and evaluation of a large scale webbased error reporting system. Qual Saf Health Care 2007; 16(04):297-302

31 Glaser BG, Stauss AL. The Discovery of Grounded Theory. Chicago: Aldine; 1967

32 Lincoln Y, Guba E. Naturalistic Inquiry. Beverly Hills, CA: Sage Publications Inc.; 1985:334-341

33 Canadian Nurses Association. Framework for the practice of registered nurses in CANADA; 2015. Available at: https://www. cna-aiic.ca/ /media/cna/page-content/pdf-en/framework-forthe-pracice-of-registered-nurses-in-canada.pdf?la=en. Accessed April 16, 2018

34 Alexander GL, Madsen RW, Miller EL, Wakefield DS, Wise KK, Alexander RL. The state of nursing home information technology sophistication in rural and nonrural US markets. J Rural Health 2017;33(03):266-274

35 Alexander GL, Madsen RW, Miller EL, et al. A national report of nursing home information technology: year 1 results. J Am Med Inform Assoc 2017;24(01):67-73

36 Abramson EL, McGinnis S, Moore J, Kaushal R; HITEC investigators. A statewide assessment of electronic health record adoption and health information exchange among nursing homes. Health Serv Res 2014;49(1 Pt 2):361-372

37 Alexander GL, Popejoy L, Lyons V, et al. Exploring health information exchange implementation using qualitative assessments of nursing home leaders. Perspect Health Inf Manag 2016;13(Fall):1f

38 Pillemer K, Meador RH, Teresi JA, et al. Effects of electronic health information technology implementation on nursing home resident outcomes. J Aging Health 2012;24(01):92-112

39 Kruse CS, Mileski M, Vijaykumar AG, Viswanathan SV, Suskandla $\mathrm{U}$, Chidambaram Y. Impact of electronic health records on longterm care facilities: systematic review. JMIR Med Inform 2017; 5(03):e35

40 Alexander GL, Rantz M, Galambos C, et al. Preparing nursing homes for the future of health information exchange. Appl Clin Inform 2015;6(02):248-266

41 Alexander GL, Steege LM, Pasupathy KS, Wise KK. Case studies of IT sophistication in nursing homes: a mixed method approach to examine communication strategies about pressure ulcer prevention practices. Int J Ind Ergon 2015;(49):156-166

42 Alexander GL, Madsen D, Herrick S, Russell B. Measuring IT Sophistication in Nursing Homes. Advances in Patient Safety: New Directions and Alternative Approaches (Vol. 4: Technology and Medication Safety). Rockville, MD: Agency for Healthcare Research and Quality (US); 2008 August. Available at: https://www.ncbi.nlm. nih.gov/books/NBK43764/. Accessed April 16, 2018 